\title{
REGULAR ELEMENTS IN ALGEBRAIC GROUPS OF PRIME CHARACTERISTIC
}

\begin{abstract}
BRIAN PARSHALL ${ }^{1}$
Abstract. A result of Steinberg's on the existence of rational regular unipotent elements in quasi-split simple algebraic groups over fields of arbitrary characteristic is partially extended to the case of non-quasi-split groups.
\end{abstract}

1. Introduction. Let $k$ be a field of characteristic $p$ and let $G$ be a simple algebraic group defined over $k$. Let $S$ be a maximal $k$-split torus of $G$ and put $d=\operatorname{dim} Z_{G}(S)$. An element of $G_{k}$ is called $k$-split if it belongs to the $k$-split radical $S \cdot{ }_{k} U$ of a minimal $k$-parabolic subgroup $P=Z_{G}(S)$. ${ }_{k} U$ of $G$. Such an element $g$ is called $k$-regular if $\operatorname{dim} Z_{G}(g)=d$. The purpose of this paper is to prove the theorem below which gives conditions under which $k$-regular unipotent elements exist. The case when $G$ is quasi-split over $k$ has been dealt with by Steinberg [10].

THeORem. Assume $G$ is not quasi-split, but $d<\operatorname{dim} G$. If the root system ${ }_{k} \Sigma$ of $S$ in $G$ is reduced, $k$-regular unipotent elements exist provided $p$ is not one of the following types for the given ${ }_{k} \Sigma: A_{r}, p \mid(r+1) ; B_{r}, p=2$; $C_{r}, p \mid 2 r ; F_{4}, G_{2}, p=2$, 3. If ${ }_{k} \Sigma$ is of type $B C_{r}, k$-regular unipotent elements exist provided $p \nmid 2(2 r+1)$ and for $\alpha, 2 \alpha \in{ }_{k} \Sigma$ there exists a $k$-split 3dimensional simple subgroup $K$ of $G$ normalized by $S$ with $\pm \alpha \mid(K \cap S)$ the roots of $K \cap S$ in $K$.

Remarks. (1) If $p$ is not "bad" for $G$ in the sense of Springer [8], every unipotent element of $G_{k}$ is $k$-split. See [9, p. 185].

(2) We do not know if our conditions on $p$ can be dropped. Various examples indicate they perhaps can be.

(3) For an indication of when $K$ exists see [7, pp. 121-125].

The proof is given in $\S 3$ below. $\S 2$ contains some elementary preliminaries on representations. Unexplained notation is that of [2].

2. Preliminaries. Let $\Sigma$ be a simple (reduced) root system in Euclidean space $E, \Delta$ a fundamental system defined by a linear order on $E$. Let

Received by the editors July 20, 1972 .

AMS (MOS) subject classifications (1970). Primary 20G15.

Key words and phrases. Simple algebraic group, $k$-regular unipotent element, split simple subgroup.

${ }^{1}$ Research supported by NSF Grant GP-29081.

(c) American Mathematical Society 1973 
$\mathscr{D} \subset E$ be the set of dominant weights defined by $\Delta$. In addition to the linear order, we partially order $E$ by $\omega>\omega^{\prime} \Leftrightarrow \omega-\omega^{\prime}$ is a sum of positive roots. Let $\Sigma_{S}$ denote the set of short roots in $\Sigma$ (with $\Sigma_{S}=\varnothing$ if there is only one root length). Put $\Delta_{S}=\Delta \cap \Sigma_{S}, s=\left|\Delta_{S}\right|, r=|\Delta|$.

Let $H$ be the simple simply connected $k$-split algebraic group with root system $\Sigma$ relative to a fixed maximal $k$-split torus $S$. Let $\omega \in \mathscr{D}$ and let $\rho: H \rightarrow G L(\mathscr{V})$ be the irreducible $k$-rational representation of dominant weight $\omega$. We are interested in the $\omega$ which have the properties that (1) the $\omega^{\prime}<\omega$ in $\mathscr{D}$ form an ordered sequence $\omega=\omega_{1}>\omega_{2}>\cdots>\omega_{n}$, and (2) there is a unique sequence $\left\{\alpha_{1}, \cdots, \alpha_{m_{2}}, \cdots, \alpha_{m_{3}}, \cdots, \alpha_{m_{n}}=\alpha_{m}\right\}$ of roots in $\Delta$ such that for $t \leqq m, \omega-\sum_{j=1}^{t} \alpha_{i}$ is conjugate under the Weyl group $W$ of $\Sigma$ to one of the $\omega_{j}$ and equals $\omega_{h}$ for $t=m_{h}$. The following result summarizes some facts we will use.

Proposition 1. Suppose $\omega$ satisfies (1) and (2) above. Assume also each $\omega_{i}$ is a weight of $S$ in $\mathscr{V}$. Let $\rho^{\prime}: H \rightarrow G L\left(\mathscr{V}^{\prime}\right)$ be a rational representation of $H$. Let $0 \neq v \in \mathscr{V}^{\prime}$ be of weight $\omega$ and fixed by $U$, the unipotent radical $R_{u}(B)$ of the Borel subgroup $B$ defined by $S$ and $\Delta$. Let $\mathscr{U}$ be the submodule of $\mathscr{V}^{\prime}$ generated by $v$. Then if $\mathscr{W}$ is a proper $H$-stable subspace of $\mathscr{U}, H$ acts trivially on $\mathscr{W}^{\prime}$. Secondly, suppose that if $0 \neq v^{\prime} \in \mathscr{V}^{\prime}$ has weight $\omega^{\prime} \in \mathscr{D}$ and is fixed by $U$, then $\omega^{\prime}=\omega_{i}$, some $i$. Let $\mathscr{Z}=\left\{z \in \mathscr{V}^{\prime}: \rho(H) z=z\right\}$, $\mathscr{V}^{\prime \prime}=\mathscr{V}^{\prime} \mid \mathscr{Z}$, and let $\rho^{\prime \prime}: H \rightarrow G L\left(\mathscr{V}^{\prime \prime}\right)$ be the induced representation. Then $\rho^{\prime \prime}$ is completely reducible.

Proof. Let $B^{-}$be the Borel subgroup defined by $S$ and $-\Delta$, and put $U^{-}=R_{u}\left(B^{-}\right)$. Then $S \cdot U^{-} \cdot U$ is dense in $H$, so $\mathscr{U}=k \rho^{\prime}(H) v=\bar{k} \rho^{\prime}\left(S \cdot U^{-}\right) v$. Hence $\omega_{1}=\omega$ is the dominant weight of $S$ in $\mathscr{U}$, and taking $\mathscr{W}$ to be maximal proper $H$-stable, $\mathscr{U} / \mathscr{W}$ is equivalent to $\mathscr{V}$. Since the $\omega_{i}$ are weights of $S$ in $\mathscr{V}$, they are weights of $S$ in $\mathscr{U}$. If $H$ does not act trivially on $\mathscr{W}$, it follows from condition (1) on $\omega$ that the dominant weight of $S$ in $\mathscr{W}$ equals some $\omega_{j}$ and so the corresponding weight space $\mathscr{U}_{\omega_{j}}$ of $\mathscr{U}$ has dimension $>1$. But let $u=u_{-\alpha_{m_{j}}} \cdots u_{-\alpha_{1}}$ where $1 \neq u_{-\alpha_{i}} \in U_{-\alpha_{i}}$. Then if $\pi$ is the projection of $\mathscr{U}$ onto $\mathscr{U}_{\omega_{j}}, \pi\left(\rho^{\prime}(u) v\right)$ must span $\mathscr{U}_{\omega_{j}}$ by condition (2) on $\omega$ and Lemme 1, Exposé 21 [3]. Hence, $H$ acts trivially on $\mathscr{W}$. For the second assertion of the proposition, we first remark that Lemme 1, Exposé 21 [3] shows that 0 is the only element of $\mathscr{V}^{\prime \prime}$ fixed by $H$. Let $\left\{v_{1 j}\right\}_{j \in J_{1}}$ be a basis for the $\omega_{1}$-weight space $\mathscr{V}_{\omega_{1}}^{\prime \prime}$ of $\mathscr{V}^{\prime \prime}$. Then $\mathscr{V}_{1 j}^{\prime \prime} \bar{k} \rho^{\prime \prime}(H) v_{1 j}$ is irreducible of dominant weight $\omega_{1}$ by above, and the sum $\mathscr{V}_{1}^{\prime \prime}=\sum_{j \in J_{1}} \mathscr{V}_{1 j}^{\prime \prime}$ is direct. Let $\omega_{t}$ be the next member of the sequence $\omega_{1}>\omega_{2}>\cdots>\omega_{n}$ for which there exists $0 \neq v^{\prime \prime} \in \mathscr{V}_{\omega_{t}}^{\prime \prime}$ with $\rho^{\prime \prime}(U) v^{\prime \prime}=v^{\prime \prime}$. Let $\left\{v_{2 j}\right\}_{j \in J_{2}}$ be maximal linearly independent in $\mathscr{V}_{\omega_{t}}^{\prime \prime}$ with $\rho^{\prime \prime}(U) v_{2 j}=v_{2 j}$ all $j$. Then $\mathscr{V}_{2 j}^{\prime \prime}=\bar{k} \rho^{\prime \prime}(H) v_{2 j}$ is irreducible of dominant weight $\omega_{t}$ and the sum $\mathscr{V}_{2}^{\prime \prime}=\mathscr{V}_{1}^{\prime \prime}+\sum_{j \in J_{2}} \mathscr{V}_{2 j}^{\prime \prime}$ is direct. Continuing in this way we obtain in $q$ 
steps, say, a completely reducible submodule $\mathscr{V}_{q}^{\prime \prime}$ of $\mathscr{V}^{\prime \prime}$ with the property that if $v^{\prime \prime} \in \mathscr{V}^{\prime \prime}$ has weight $\omega^{\prime} \in \mathscr{D}$ and $\rho^{\prime \prime}(U) v^{\prime \prime}=v^{\prime \prime}$ then $v^{\prime \prime} \in \mathscr{V}_{q}^{\prime \prime}$. Hence $H$ acts trivially on $\mathscr{V}^{\prime \prime} \mid \mathscr{V}_{q}^{\prime \prime}$, whence $\mathscr{V}^{\prime \prime}=\mathscr{V}_{q}^{\prime \prime}$, proving the proposition. We also note that in the first part of the proof we have shown that if $\lambda$ is a nonzero weight in $\mathscr{V}$, then $\operatorname{dim} \mathscr{V}_{\lambda}=1$ since $\lambda$ is conjugate to some $\omega_{i}$.

We assume familiarity with the construction of the irreducible representations of $H$ from those of the corresponding complex simple Lie algebra by "reduction mod $p$ " $[\mathbf{1 1}, \S 12]$. For the remainder of this section we determine conditions on $p$ for which certain modules and maps remain irreducible and nonsingular in passage from characteristic 0 to characteristic $p$. Let $\mathscr{L}$ be the complex simple Lie algebra with root system $\Sigma$, and let $\mathscr{U}$ be the universal enveloping algebra for $\mathscr{L}$. Let $\left\{X_{\alpha}, H_{\beta}: \alpha \in \Sigma\right.$, $\beta \in \Delta\}$ be a Chevalley basis for $\mathscr{L}_{[11}$, p. 6], and let $\mathscr{U}_{\boldsymbol{Z}}, \mathscr{U}_{\boldsymbol{Z}}^{+}, \mathscr{U}_{\boldsymbol{Z}}^{-}$be the $Z$-subalgebras of $\mathscr{U}$ generated by $X_{\alpha}^{m} / m !\left(m \in Z^{+}\right)$for $\alpha \in \Sigma, \Sigma^{+}, \Sigma^{-}$, respectively. Let $\mu$ be the maximal root in $\Sigma$, and if $\Sigma_{S} \neq \varnothing$ let $v$ be the maximal short root. For convenience we agree in the case of $A_{1}$ that $\mu$ is both long and short (so $\mu=v$ ). In the following we take $\omega \in \mathscr{D}$ to be one of three possibilities: $\omega=\mu, \nu$, or in the case when $\Sigma$ is of type $B_{r}$ we allow $\omega=2 v$. Conditions (1) and (2) preceding Proposition 1 are easily verified for these $\omega$. Let $\mathscr{V}^{c}$ be the irreducible $\mathscr{L}$-module of dominant weight $\omega$. For $0 \neq v \in \mathscr{V}_{\omega}^{C}, M=\mathscr{U}_{Z}^{-} v$ is a $\mathscr{U}_{Z}$-stable lattice in $\mathscr{V}^{C}$ [11, p. 17]. For a nonzero weight $\lambda$ of $\mathscr{L}$ in $\mathscr{V}^{c}$, let $v_{\lambda} \in M$ be so that $Z v_{\lambda}=M \cap \mathscr{V}_{\lambda}^{C}$. Let $\Sigma^{\prime}=\left\{\alpha \in \Sigma: \alpha\right.$ is a weight in $\left.\mathscr{V}^{C}\right\}=\Sigma$ or $\Sigma_{S}$. Let $M_{0}^{\prime} \subset M \cap \mathscr{V}_{0}^{C}$ be the $Z$ span of the $X_{-\gamma} v_{\gamma}$ for $\gamma \in \Delta^{\prime}=\Delta \cap \Sigma^{\prime}$. The nonzero weights in $\mathscr{V}^{C}$ are roots and, if $\omega=2 v$, twice short roots. Thus, for $\alpha \neq \beta$ in $\Delta^{\prime}, X_{-\beta} X_{\beta} v_{\alpha}=$ $-\alpha\left(H_{\beta}\right) v_{\alpha}$. Hence, once for a given $\gamma \in \Delta^{\prime}, v_{\gamma}$ is fixed (it is unique up to changes in sign), since $M$ is $\mathscr{U}_{Z^{-}}$stable, the other $v_{\beta}, \beta \in \Delta^{\prime}$, are uniquely determined such that for $\alpha, \beta \in \Delta^{\prime}, X_{\beta} X_{-\alpha} v_{\alpha}=\langle\alpha, \beta\rangle v_{\beta}$ where $\langle\alpha, \beta\rangle=$ $\alpha\left(H_{\beta}\right)$ or $\beta\left(H_{\alpha}\right)$ (with $\langle\beta, \alpha\rangle$ then equal to $\beta\left(H_{\alpha}\right)$ or $\alpha\left(H_{\beta}\right)$, respectively), except if $\omega=2 v$ and $\alpha=\beta \in \Sigma_{S}$ when $\langle\alpha, \alpha\rangle=6$ because of the representation theory of the simple Lie algebra $\mathrm{C} X_{\alpha}+\mathrm{CH}_{\alpha}+C X_{-\alpha}$ acting irreducibly on the 5-dimensional space it generates from $v_{2 \alpha}$ [4, p. 83]. Given $v=$ $\sum_{\alpha \in \Delta^{\prime}} c_{\alpha} X_{-\alpha} v_{\alpha} \in \bar{k} \otimes M_{0}^{\prime}$, in order that $X_{\beta} v=0$ for all $\beta \in \Delta^{\prime}$ we must therefore have $\sum_{\alpha \in \Delta^{\prime}} c_{\alpha}\langle\alpha, \beta\rangle=0, \beta \in \Delta^{\prime}$. The determinant of the matrix $C^{\prime}$ of this 'system of equations is by above the determinant of the Cartan matrix of $\Delta^{\prime}$, except if $\omega=2 v$ when it is easily calculated to be $2(2 r+1)$.

Consider the irreducible $H$-module $\mathscr{V}$ of dominant weight $\omega$ obtained from the $\mathscr{L}$-module $\mathscr{V}^{\boldsymbol{c}}$. Given $\omega_{i}(i>1), \lambda=\omega_{i}+\alpha_{m_{i}}$ is $W$-conjugate to $\omega_{j}$ by condition (2) on $\omega$, and $\omega_{j}>\omega_{i}$ since $\lambda>\omega_{i}$. Then

$$
X_{\alpha_{m_{i}}} v_{\omega_{i}}=X_{\alpha_{m_{i}}} X_{-\alpha_{m_{i}}} v_{\lambda}=H_{\alpha_{m_{i}}} v_{\lambda}=\lambda\left(H_{\alpha_{m_{i}}}\right) v_{\lambda}
$$

since $\lambda+\alpha_{m_{i}}$ is not a weight in $\mathscr{V}^{c}$. Thus, if $p+\lambda\left(H_{\alpha_{m_{i}}}\right)(=-2$ if $i=2$ with 
$\omega=\mu$ in $B_{r}, C_{r}, F_{4}$, or if $i=2,3$ with $\omega=2 v$ in $B_{r} ;=-3$ if $i=2, \omega=\mu$ in $\left.G_{2}\right) \omega_{i}$ is a weight of $S$ in $\mathscr{V}$ by induction on $i$. If in addition $p \nmid \operatorname{det} C^{\prime}$, we have also shown $\operatorname{dim} \mathscr{V}_{0}=\left|\Delta^{\prime}\right|=r$ or $s$.

For $\alpha \in \Delta$, put $n_{\alpha}=\operatorname{Exp}\left(X_{\alpha}\right) \operatorname{Exp}\left(-X_{-\alpha}\right) \operatorname{Exp}\left(X_{\alpha}\right)$, an automorphism of $\mathscr{V}^{c}$ with $n_{\alpha} M=M$. We order $\Delta$ so that the first $q$ roots $\alpha_{1}, \cdots, \alpha_{q}$ are orthogonal as are the last $r-q$ roots. The Coxeter transformation $w \Delta$, $w_{\alpha_{r}} \cdots w_{\alpha_{1}}$ acting on $\mathscr{V}_{0}^{C}$ is given by $n_{w}=n_{\alpha_{r}} \cdots n_{\alpha_{1}}$. For $\gamma \in \Delta^{\prime}, \alpha \in \Delta$, $n_{\alpha} X_{-\gamma} v_{\gamma}=X_{-\gamma} v_{\gamma}$ if $\alpha \notin \Delta^{\prime}$ (since otherwise from the form of $n_{\alpha}$ we would have $\left.\alpha \in \Sigma^{\prime}\right)$, and $n_{\alpha} X_{-\gamma} v_{\gamma}=X_{-\gamma} v_{\gamma}-\langle\gamma, \alpha\rangle^{\prime} X_{-\alpha} v_{\alpha}$ for $\alpha \in \Delta^{\prime}$ where $\left\langle\gamma, \alpha^{\prime}\right\rangle=$ $\langle\gamma, \alpha\rangle$ if $\alpha \neq \gamma$ and $\left\langle\alpha, \alpha^{\prime}\right\rangle=2$. For $i \leqq q$

while for $i>q$,

$$
\left(1-n_{w}\right) X_{-\alpha_{i}} v_{\alpha_{i}}=2 X_{-\alpha_{i}} v_{\alpha_{i}}-\sum_{j>q ; \alpha_{j} \in \Delta^{\prime}}\left\langle\alpha_{i}, \alpha_{j}\right\rangle^{\prime} X_{-\alpha_{j}} v_{\alpha j}
$$

$$
\begin{aligned}
\left(1-n_{w}\right) X_{-\alpha_{i}} v_{\alpha_{i}} & \\
& =2 X_{-\alpha_{i}} v_{\alpha_{i}}-\sum_{j \leqq q ; \alpha_{j} \in \Delta^{\prime}}\left\langle\alpha_{i}, \alpha_{j}\right\rangle^{\prime} n_{w} X_{-\alpha_{j}} v_{\alpha_{j}} \\
& =2 X_{-\alpha_{i}} v_{\alpha_{i}}+\sum_{j \leqq q}\left\langle\alpha_{i}, \alpha_{j}\right\rangle^{\prime}\left(X_{-\alpha^{\prime}} v_{\alpha_{j}}-\sum_{k>q ; \alpha_{k} \in \Delta^{\prime}}\left\langle\alpha_{j}, \alpha_{k}\right\rangle^{\prime} X_{-\alpha_{k}} v_{\alpha_{k}}\right) .
\end{aligned}
$$

Substituting the conditions $\left({ }^{*}\right)$ into $\left({ }^{*}\right)$ we transform the matrix obtained for $1-n_{w}$ into $\left(\left|c_{i j}^{\prime}\right|\right)$ where $\left(c_{i j}^{\prime}\right)$ is the Cartan matrix of $\Delta^{\prime}$. A simple induction argument shows $\operatorname{det}\left(\left|c_{i j}^{\prime}\right|\right)=\operatorname{det}\left(c_{i j}^{\prime}\right)$.

Collecting together the various restrictions on $p$, we get

Proposition 2. Let $\omega=\mu, v$, or in the case of $B_{r}$ possibly $2 v$. For the following $\Sigma$ suppose $p$ is as indicated: $A_{r}, p \nmid(r+1) ; C_{r}, p \nmid 2 r ; B_{r}, p \neq 2$ if $\omega<2 v$ and $p+2(2 r+1)$ if $\omega=2 v ; F_{4}, G_{2}, p \neq 2$, 3 . Let $\rho: H \rightarrow G L(\mathscr{V})$ be the irreducible representation of $H$ of dominant weight $\omega$. Then if $\omega^{\prime}<\omega$, $\omega^{\prime} \in \mathscr{D}, \omega^{\prime}$ is a weight of $S$ in $\mathscr{V}$. Also, $\operatorname{dim} \mathscr{V}_{0}=r$ except if $\omega=v$ when it equals s. Finally, 1 is not an eigenvalue of $\rho\left(n_{w}\right) \mid \mathscr{V}_{0}$ if $n_{w} \in N_{H}(S)$ represents the Coxeter transformation $w$.

We also need

Proposition 3. The Coxeter transformation $w$ defined above decomposes $\Sigma$ into $r$ orbits of roots and $\Sigma_{S}$ into $s$ orbits of roots.

Proof. As is well known [5, Corollary 8.2], $w$ decomposes $\Sigma$ into $r$ orbits each with $h=1$ height $\mu$ elements. Therefore, the second assertion follows since $\left|\Sigma_{S}\right|\left(=2 r, B_{r} ; 2 r(r-1), C_{r} ; 26, F_{4} ; 6, G_{2}\right)$ divided by $h(=2 r$, $\left.B_{r}, C_{r} ; 13, F_{4} ; 6, G_{2}\right)$ equals $s\left(=1, B_{r}, G_{2} ; r-1, C_{r} ; 2, F_{4}\right)$.

3. Proof of the Theorem. Put $G_{0}^{r}=Z_{G}(S), \mathfrak{G}=\operatorname{Lie}(G), \mathfrak{G}_{0}^{r}=\operatorname{Lie}\left(G_{0}^{r}\right)=$ $Z_{\mathfrak{W}}(S)$. Let ${ }_{k} \Delta$ be a fundamental system for ${ }_{k} \Sigma$, and for $\alpha \in_{k} \Delta$, let $1 \neq u_{\alpha} \in$ $U_{(\alpha), k}$. Define $u=\prod_{\alpha_{k} \Delta} u_{\alpha}$ in some order. We assume first that the root 
system ${ }_{k} \Sigma$ of $S$ is reduced. Borel and Tits [2, §7] have constructed a $k$ split simple subgroup $H \supset S$ such that the $u_{\alpha} \in H$, and so that the root system $\Sigma_{H}$ of $S$ in $H$ identifies with ${ }_{k} \Sigma$. Let $\rho: H \rightarrow G L(\mathfrak{G})$ be the adjoint representation of $H$ in $\mathfrak{5}$. Let $\mathscr{Z}=Z_{\mathfrak{G}}(H) \subset \mathfrak{F}_{0}^{r}$, and put $\mathscr{G}^{\prime}=\mathfrak{G} / \mathscr{Z}$ with $\rho^{\prime}: H \rightarrow G L\left(\mathfrak{G}^{\prime}\right)$ the induced representation. We restrict $p$ to not being one of the types listed in the theorem, and Propositions 1 and 2 show $\rho^{\prime}$ is completely reducible. Let $d_{\mu}$ and $d_{v}$ be the dimensions respectively of $U_{(\mu)}$ and $U_{(v)}$. Then $\left(5\right.$ decomposes as $\mathscr{V}_{1} \oplus \mathscr{V}_{2}$ where $\mathscr{V}_{1}$ (resp. $\mathscr{V}_{2}$ ) is the direct sum of $d_{\mu}$ (resp. $d_{v}-d_{\mu}$ ) irreducible $H$-submodules of dominant weight $\mu($ resp. $v)$. It is clear that $\operatorname{dim} Z_{\mathfrak{G}}(u) \leqq \operatorname{dim} Z_{\mathfrak{G}^{\prime}}(u)+\operatorname{dim} \mathscr{Z}$. Since $\operatorname{dim} Z_{\mathfrak{G}}(u) \geqq \operatorname{dim} Z_{G}(u) \geqq d$ trivially, it suffices to show $\operatorname{dim} Z_{\mathfrak{W}^{\prime}}(u)+$ $\operatorname{dim} \mathscr{Z} \leqq d$. We have $Z_{\mathfrak{F}^{\prime}}(u)=Z_{\mathscr{V}_{1}}(u) \oplus Z_{\mathscr{V}_{2}}(u)$. Let $\mathscr{W}_{1}$ and $\mathscr{W}_{2}$ be irreducible $H$-modules of dominant weights $\mu$ and $\nu$ respectively. By Proposition 2 if $n_{w} \in H$ represents the Coxeter transformation defined earlier, $1-n_{w}$ is nonsingular on the zero weight spaces $\left(\mathscr{W}_{i}\right)_{0}$. By [10, Lemma 4.5], $u$ is conjugate in $H$ to an element $y$ of the form $b n_{w}, b \in B$. By Proposition $3, w$ decomposes ${ }_{k} \Sigma$ into $r=\left.\right|_{k} \Delta \mid$ orbits of roots and ${ }_{k} \Sigma_{S}$ into $s=\left|{ }_{k} \Delta_{S}\right|$ orbits of roots, so that exactly as in [10, Lemma 4.3], $\operatorname{dim} Z_{\mathscr{W}_{1}}(y) \leqq r$ and $\operatorname{dim} Z_{\mathscr{W}_{2}}(y) \leqq s$. Hence, $\operatorname{dim} Z_{\mathscr{V}_{1}}(u) \leqq d_{\mu} r$ and $\operatorname{dim} Z_{\mathscr{V}_{2}}$ $(u) \leqq\left(d_{v}-d_{\mu}\right) s$. By Proposition 2, $r=\operatorname{dim}\left(\mathscr{W}_{1}\right)_{0}$ and $s=\operatorname{dim}\left(\mathscr{W}_{2}\right)_{0}$, so $\operatorname{dim} Z_{\mathfrak{G}^{\prime}}(u) \leqq d_{\mu} r+\left(d_{v}-d_{\mu}\right) s=\operatorname{dim} Z_{\mathfrak{G}^{\prime}}(S)$. Since $\mathscr{Z} \subset \mathfrak{G}_{0}^{r}$ we get finally $\operatorname{dim} Z_{\mathfrak{W}^{\prime}}(u)+\operatorname{dim} \mathscr{Z} \leqq \operatorname{dim} \mathfrak{G}_{0}^{r}=d$, as desired.

Next, assume ${ }_{k} \Sigma$ is of type $B C_{r}, P \nmid 2(2 r+1)$, and the subgroups $K$ exist. Then [6, Proposition 5.1], there exists a simple $k$-split subgroup $H \supset S$ of type $B_{r}$. Put $d_{\mu}=\operatorname{dim} U_{(\mu)}, d_{v}=\operatorname{dim} U_{(v)} / U_{(2 v)}, d_{2 v}=\operatorname{dim} U_{(2 v)}$. Define $u$ as before with $u \in H$. Let $\rho_{i}: H \rightarrow G L\left(\mathscr{W}_{i}\right), i=1,2,3$, be irreducible of dominant weight $2 v, \mu, v$, respectively. $H$ acts completely reducibly on. the $\mathfrak{G F}^{\prime}$ defined as above, and $\mathfrak{G}^{\prime}=\mathscr{V}_{1} \oplus \mathscr{V}_{2} \oplus \mathscr{V}_{3}$ where $\mathscr{V}_{i}$ is the direct sum of $c_{i}$ copies of $\mathscr{W}_{i}\left(c_{1}=d_{2 v}, c_{2}=d_{\mu}-d_{2 v}, c_{3}=d_{v}-d_{\mu}\right)$. As before, $\operatorname{dim} Z_{\mathscr{W}_{2}}(u) \leqq r$ and $\operatorname{dim} Z_{\mathscr{W}_{3}}(u) \leqq s$, so to finish we must show $\operatorname{dim} Z_{\mathscr{W}_{1}}(u) \leqq$ $r$. Put ${ }_{k} \Delta=\left\{\alpha_{1}, \cdots, \alpha_{r}\right\}$ with $\alpha_{i}$ connected to $\alpha_{i+1}(i<r), \alpha_{1}$ long, $\alpha_{r}$ short. If $\lambda=\sum n_{i} \alpha_{i} \in Z_{k} \Delta$, call $h(\lambda)=\sum n_{i}$ the height of $\lambda$. Let $\left(\mathscr{W}_{1}\right)_{i}$ be the subspace of $\mathscr{W}_{1}$ spanned by vectors of weight of height $i$, and let $\pi_{i}$ be the projection of $\mathscr{W}_{1}$ onto $\left(\mathscr{W}_{1}\right)_{i}$. We say a weight $\alpha^{\prime}$ is related to a weight $\lambda$ if $\lambda=\lambda^{\prime}+\alpha$ for some $\alpha \in_{k} \Delta$. If $\lambda^{\prime}$ is so related to $\lambda \neq 0$, since $p \neq 2$, a slight extension of the argument on p. 6 (or Proposition 2.2, [6]) shows $\pi_{\lambda} \rho_{1}(u)$ $\left(\mathscr{W}_{1}\right)_{\lambda^{\prime}}=\left(\mathscr{W}_{1}\right)_{\lambda}, \pi_{\lambda}$ the projection of $\mathscr{W}_{1}$ onto $\left(\mathscr{W}_{1}\right)_{\lambda}$. Define $\beta_{i}=\alpha_{i}$ if $1 \leqq i \leqq r$ and $=\alpha_{2 r-i+1}$ if $r<i \leqq 2 r$. For $1 \leqq j \leqq 2 r$, put $\gamma_{j, t}=\beta_{t}+\cdots+$ $B_{j+t-1}$ for $1 \leqq t \leqq \eta_{j}=\min \{r, r-j+2\}$, the weights of heights $j$. If $j>1$, $\gamma_{j-1,1}$ is related only to $\gamma_{j, 1}$ and if $t>1, \gamma_{j-1, t}$ is related only to $\gamma_{j, t-1}$ and $\gamma_{j, t}\left(\right.$ if $\left.t \leqq \eta_{j}\right)$. Hence, if $j>1, \pi_{j} \rho_{1}(u)$ maps $\left(\mathscr{W}_{1}\right)_{j-1}$ onto $\left(\mathscr{W}_{1}\right)_{j}$. Similarly, if $-1 \geqq j>-2 r, \pi_{j} \rho_{1}(u)$ maps $\left(\mathscr{W}_{1}\right)_{j-1}$ injectively into $\left(\mathscr{W}_{1}\right)_{j}$. The proof 
that $\operatorname{dim}\left(\mathscr{W}_{1}\right)_{0}=r$ (Proposition 2) shows $\pi_{j} \rho_{1}(u)$ maps $\left(\mathscr{W}_{1}\right)_{j-1}$ injectively into $\left(\mathscr{W}_{1}\right)_{j}, j=0,1$. Hence, $\operatorname{dim} Z_{\mathscr{W}_{1}}(u) \leqq \sum_{j \geqq 1}\left(\operatorname{dim}\left(\mathscr{W}_{1}\right)_{j}-\operatorname{dim}\left(\mathscr{W}_{1}\right)_{j+1}\right)=$ $\operatorname{dim}\left(\mathscr{W}_{1}\right)_{1}=r$, as desired.

Finally, we should note the characteristic zero case is much simpler, a $k$-regular unipotent element being obtained by taking the exponential of a $k$-principal nilpotent element in $\mathfrak{6}$. See [1] and [5].

\section{BIBLIOGRAPHY}

1. P. Andre, R-regular elements in semi-simple algebraic groups, Thesis, Yale University, New Haven, Conn., 1970.

2. A. Borel and J. Tits, Groupes réductifs, Inst. Hautes Études Sci. Publ. Math. No. 27 (1965), 55-150. MR 34 \#7527.

3. C. Chevalley, Classification des groupes de Lie algébriques. Vols. I, II, Hermann, Paris, 1956, 1958.

4. N. Jacobson, Lie algebras, Interscience Tracts in Pure and Appl. Math., no. 10, Interscience, New York, 1962. MR 26 \#1345.

5. B. Kostant, The principal three-dimensional subgroups and the Betti numbers of a complex simple Lie group, Amer. J. Math. 81 (1959), 973-1032. MR 22 \#5693.

6. B. Parshall, Rational representations and subgroups of algebraic groups, Thesis, Yale University, New Haven, Conn., 1971.

7. G. Seligman, Modular Lie algebras, Ergebnisse der Mathematik und ihrer Grenzgebiete, Band 40, Springer-Verlag, New York, 1967. MR 39 \#6933.

8. T. A. Springer, Some arithmetic results on semi-simple Lie algebras, Inst. Hautes Études Sci. Publ. Math. No. 30 (1966), 115-141. MR 34 \#5993.

9. T. A. Springer and R. Steinberg, Conjugacy classes, Lecture Notes in Math., no. 131, Springer-Verlag, New York, 1970.

10. R. Steinberg, Regular elements of semisimple algebraic groups, Inst. Hautes Études Sci. Publ. Math. No. 25 (1965), 49-80. MR 31 \#4788.

11. — Lectures on Chevalley groups, Yale University, New Haven, Conn., 1968 (mimeographed).

Department of Mathematics, University of Virginia, Charlottesville, Virginia 22903 